\title{
Féeries
}

Études sur le conte merveilleux, XVII ${ }^{-}$XIXe siècle

Le conte, les savoirs

\section{Tiphaigne de la Roche entre science et merveille}

Savoir alchimique et « alchimie du verbe » dans Amilec, ou la graine d'hommes (1753)

Tiphaigne de la Roche between Science and Marvels: Alchemical Knowledge and "alchimie du verbe", in Amilec, ou la graine d'hommes (1753)

\section{Emmanuelle Sempère}

\section{(2) OpenEdition}

\section{Journals}

Édition électronique

URL : http://journals.openedition.org/feeries/706

DOI : $10.4000 /$ feeries.706

ISSN : 1957-7753

Éditeur

UGA Éditions/Université Grenoble Alpes

Édition imprimée

Date de publication : 1 juillet 2009

Pagination : 117-130

ISBN : 978-2-84310-140-3

ISSN : $1766-2842$

\section{Référence électronique}

Emmanuelle Sempère, «Tiphaigne de la Roche entre science et merveille », Féeries [En ligne], 6 | 2009,

mis en ligne le 15 septembre 2010, consulté le 08 septembre 2020. URL : http://

journals.openedition.org/feeries/706 ; DOI : https://doi.org/10.4000/feeries.706

\section{(c) Féeries}




\title{
TIPHAIGNE DE LA ROCHE ENTRE SCIENCE ET MERVEILLE
}

\author{
SAVOIR ALCHIMIQUE ET «ALCHIMIE DU VERBE » DANS
}

AMILEC, OU LA GRAINE D'HOMMES (I753)

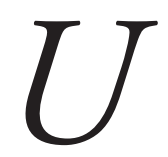

NE LECTURE ALCHIMIQUE de l'œuvre de Tiphaigne de la Roche sourd dans de nombreux commentaires, mais elle semble davantage dépendre des positions propres des critiques que de l'analyse de ses contenus; l'œuvre est en effet difficile à appréhender du fait d'une écriture volontiers anagrammatique, de l'alternance des tons sérieux et comiques, de la superposition et du mélange des savoirs, de la pauvreté enfin des données biographiques disponibles. Médecin, diplômé de la faculté de Caen, Tiphaigne n'a rien, a priori, d'un conteur fantaisiste. Mais il semble avoir passé plus de temps à écrire et à se passionner pour l'alchimie (à s'y essayer ?) qu'à exercer la médecine.

Concernant Amilec, voici l'avis d'un fort partial lecteur, l'alchimiste moderne Fulcanelli :

[c'est] un petit livre très curieux et si habilement fermé qu'il est impossible de savoir quel en est le sujet. [...] il porte ce titre singulier Amilec, ou la graine d'hommes. C'est un assemblage de l'anagramme et du calembour. Il faut lire Alcmie ou la crème d'Aum. Les néophytes apprendront que c'est là un véritable traité d'alchimie, parce que l'on écrivait, au XIII ${ }^{\mathrm{e}}$ siècle, Alkimie, alkemie, alkmie ; que le point de science révélé par l'auteur se rapporte à l'extraction de l'esprit enclos dans la matière première, ou vierge philosophique, qui porte le même signe que la Vierge céleste, le monogramme AUM ; qu'enfin cette extraction doit se faire par un procédé analogue à celui qui permet de séparer la crème du lait [...]. En ôtant le voile du titre qu'il recouvre, on voit combien celui-ci est suggestif, puisqu'il annonce la divulgation du moyen secret, propre à l'obtention de cette crème du lait de vierge, que peu de chercheurs ont eu le bonheur de posséder ${ }^{\mathrm{r}}$.

I. Fulcanelli, Les Demeures philosophales [1930], Paris, 1965, préf. E. Canseliet, t. I, p. I08. Fulcanelli, figure respectée de l'alchimie moderne, admiré d'A. Breton, a gardé secrète son identité ; il voyait en Cyrano de Bergerac " un philosophe hermétique d'exceptionnelle valeur " (ibid., p. 50).

Féeries, nº, 2009, p. II7-I30. II7

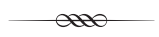


Jacques Marx, auteur de l'étude de référence sur Tiphaigne de la Roche, écarte cette interprétation "peu solide " en invoquant non le détail du texte, mais son genre et son sujet : ce serait avant tout " une satire " de l'humanité, le thème de la génération correspondant à une " nouvelle distanciation réduisant l'humanité à ses éléments constitutifs les plus simples et les plus représentatifs, les germes, qui contiennent toutes les déterminations de l'univers social ${ }^{2} »$. Dès lors, on voit bien le point de discussion : le " détail » du texte résiste à l'interprétation, en imposant au lecteur de paradoxales associations d'idées et de termes.

Amilec, ou la graine d'hommes est un petit roman paru anonymement en $1753^{3}$; le narrateur raconte qu'un génie, Amilec, lui est apparu en songe pour lui expliquer le phénomène de la génération et le peuplement des planètes. Une "génération " pensée sur le modèle de la préformation : des " graines ", échappées ou récoltées par des génies, sélectionnées, semées puis cultivées dans l'univers. Génération qui tout à la fois souligne et répare la médiocrité humaine : de là la tournure satirique du texte, revue d'étourdis et de coquettes. Régénération aussi, qui tient à la fois du discours moral, de la réforme politique, de la science naturelle et de l'alchimie.

\section{Génération, dégénérescence et régénération}

Les références scientifiques du texte sont non seulement sérieuses et nombreuses, mais vivantes: Tiphaigne compile et discute les savoirs de son temps sur la génération, illustrant avec Amilec les hypothèses préformationnistes (inspirées par Leeuwenhoek en particulier) ainsi que la théorie des affinités, formulée en I7I8 par Geoffroy ${ }^{4}$. Mais Tiphaigne présente moins un "système " que des " possibles " de la génération, déclinés à partir de principes communs - il y aurait, à la base de toute génération, une " graine " d'hommes, c'est-à-dire une semence contenant en petit des éléments préformés d'individus humains, et une "matrice ", conçue comme le " lieu " de la fécondation. La "graine » elle-même est créée par "végétation », soit par la rencontre entre un «tubule » et un « levain », à la suite d'un processus de fermentation (p. 87) qui apparaît comme l'un des

2. Jacques Marx, Tiphaigne de la Roche : modèles de l'imaginaire au XVIII siècle, Université libre de Bruxelles, éd. U. de Bruxelles, 198I, p. 45.

3. C.-F. Tiphaigne de la Roche, Amilec, ou la graine d'hommes, rééd. augmentée de 1754, éd. moderne, Montpellier, Grèges, 20or.

4. E. F. Geoffroy, Dissertation sur la génération de l'homme par les vers spermatiques, 1704. 
points les plus ambigus du texte. En effet, la notion est au carrefour des modèles de savoir, comme l'explique Venel dans l'Encyclopédie:

La fermentation a été dans la doctrine chimique $\&$ médicinale du siècle dernier, ce qu'a été dans la Physique la matière subtile, \& ce qu'est aujourd'hui l'attraction : elle eut aussi le même sort que l'agent cartésien, que la qualité newtonienne, \& en général que tous les principes philosophiques les plus solidement établis's.

Dans un deuxième article, beaucoup plus long, d'Aumont dénonce point par point les erreurs des "fermentateurs ", ces successeurs de Van Helmont, qu'il appelle également " humoristes " - l'ironie voulant que ce terme ait été employé par Van Helmont pour désigner ses adversaires galénistes... Tiphaigne semble adopter la même position critique, en précisant " qu'il ne faut pas confondre [l'" espèce de fermentation " dont il est question] avec celle des Chimistes " (p. 86). Cohérente semble alors la place de Van Helmont dans la galerie comique constituée par le " cabinet de curiosités » du Physicien lunaire Ataman, où les " gas " succèdent à la pierre philosophale (p. 6o).

L'analogie entre le végétal et l'animal qui fonde le système d'Amilec possède la même ambivalence foncière, en ce sens que si elle peut évoquer les correspondances alchimiques, elle renvoie surtout aux écrits de Maupertuis et de Buffon - qui certes n'écrivent pas des contes. Tiphaigne par ce biais rapporte la variété des modalités de la rencontre entre la graine et la matrice, non plus à la richesse de la Nature, mais à l'habileté d'Amilec et de ses génies. Audace fictionnelle et idéologique, qui consiste à imaginer une véritable entreprise de "sélection » et de « culture » des "germes " les plus " purs ». Nous voilà pris dans une sorte de fantasme de régénération de l'espèce humaine, déclinée en même temps comme eugénisme et comme réforme politico-sociale, réintégration mystique et progrès " naturel » de l'espèce. Si le concept d'eugénisme n'apparaît qu'à la fin du XIX ${ }^{\mathrm{e}}$ siècle avec Galton, l'idée en est très ancienne, souvent liée à d'amères considérations sur l'être humain. Pour contrecarrer une dégénérescence dont l'idée parcourt le siècle, Vandermonde ${ }^{6}$ par exemple prône le métissage et veut le promouvoir par la loi. Buffon de son côté imagine une époque heureuse où les hommes pouvaient vivre près de 9ro ans : " la surface de la Terre étant moins compacte, moins sèche, tout ce qu'elle produisait, devait être plus ductile, plus souple, plus susceptible d'extension »; il est probable

5. Encyclopédie, article «fermentation (chimie) »; l'article suivant répond à l'entrée «fermentation (économie animale)».

6. Charles-Augustin Vandermonde, Essai sur la manière de perfectionner l'espèce humaine, Paris, I756 ; voir le numéro spécial de la revue Vesalius, vol. II, nº 2, 2005. 
donc que «la durée de la vie de l'homme ait diminué peu à peu à mesure que la surface de la Terre a pris plus de solidité par l'action continuelle de la pesanteur ${ }^{7}$ ".

À cette intrication de la morale et de la science naturelle, Tiphaigne associe l'alchimie et son concept de régénération du cosmos. Dans les procédés utilisés par Amilec et ses génies, on peut ainsi reconnaître ceux de l'adepte, qui " recueille ", "purifie ", " sépare ", "sème ". L'un des textes alchimiques les plus célèbres, l'un des plus mystérieux aussi, $L a$ Table d'Emeraude, peut servir de grille à une lecture alchimique du texte de Tiphaigne : " toutes choses ont été et sont venues d'Un ${ }^{8}$ " écrit la Table, ce qui devient chez Tiphaigne " tout cela a été autrefois contenu dans un grain " (formule parfois comprise aujourd'hui comme une anticipation des conceptions modernes) - le " grain " étant en alchimie la matière de la pierre philosophale. Surtout, l'adepte est invité à « séparer la Terre du Feu, le subtil de l'épais, doucement, avec grande industrie ", ce qu'illustre chacune des opérations d'Amilec, avec des effets visuels plaisants qui semblent repris des nombreuses compilations et anthologies alchimiques, souvent abondamment illustrées, qui voient le jour aux XVII ${ }^{\mathrm{e}} \mathrm{XVIII}^{\mathrm{e}}$ siècles ${ }^{9}$. Mais cette régénération paraît bien compromise, voire totalement illusoire, dans la fiction de Tiphaigne. Amilec par exemple s'inquiète : "On dirait que la Nature s'épuise " (p. 66). Le souci de l'adepte de reproduire le processus de la Création dans son laboratoire, en accélérant les processus naturels, devient une sorte de course contre la montre pour récolter et reproduire les graines d'hommes les plus pures avant qu'elles ne disparaissent entièrement : on ne trouve presque plus de graines de "Juges irréprochables », de "filles modestes » ou d' « Ecclésiastiques [...] de bon aloi » (p. 32).

La dimension satirique achève d'ôter toute dimension mystique aux efforts d'Amilec : la " pureté » des graines est d'abord affaire de qualité sociale et d'identité (les graines de femmes par exemple, sont sujettes à faire "dégénérer » les meilleures graines masculines). Est-ce là une extrapolation du destin de la théorie de la préexistence des germes, dont la préformation n'est que le versant purement scientifique ? De fait la

7. Buffon, "De la vieillesse et de la mort ", dans Euvres, éd. de S. Schmitt, Paris, Gallimard, Bibliothèque de la Pléiade, 2007, p. 270-272.

8. La Table d'Emeraude, texte attribué à Hermès et apparu en Occident au XII siècle ; voir l'édition critique de Didier Kahn, Hermès Trismégiste, La Table d'Émeraude et sa tradition alchimique, Paris, Les Belles Lettres, 1994, p. 43 pour la présente citation (issue de la traduction française de la vulgate latine, commentée par Hortulain, $\mathrm{XIV}^{\mathrm{e}}$ siècle).

9. Voir, pour un recensement d'ouvrages et une analyse, l'article «Alchimie, Occident moderne " de Didier Kahn, dans le Dictionnaire critique de l'ésotérisme, dir. J. Servier, Paris, PUF, 1998. 
préexistence des germes appuyait la théorie sociale des qualités héréditaires, ce que Bernardin de Saint-Pierre, à la fin du siècle, dénoncera dans ses Etudes de la nature: ces lignées de rois, de nobles et de roturiers ne peuvent qu'entraver toute pensée démocratique ${ }^{10}$. L'audace de Tiphaigne sur ce plan réside dans l'éloge miné et paradoxal du duc de Bourgogne sur lequel se conclut cette galerie de types sociaux. L'épisode de « l'élection des Rois » voit Amilec et ses génies sélectionner grâce aux propriétés physiques les graines de rois les plus capables d'attirer à elles les graines de peuples - Tiphaigne s'amusant ici à fournir au lecteur une énième variation sur les tourbillons cartésiens. Le passage est l'occasion d'une revue des types de gouvernements (républicains, monarchiques, impériaux) et de l'état politique de l'Europe ; et, in fine, d'une évocation sur le mode merveilleux de la naissance du duc de Bourgogne :

[Amilec] sortit du sein des tourbillons [que font les germes de peuples entourant les germes royaux] aussi légèrement qu'un Plongeur sort du sein des eaux. "Cet auguste germe, dit-il, nous vient de l'illustre Famille des Bourbon. » (p. Io6)

Loin de la physiologie, le conte et la satire, nous voilà en pleine apothéose mythologique ; mais Amilec n'est qu'un avatar dégradé de l'AthénaMentor de Fénelon puisqu'en I753, nul n'ignore que le Dauphin, auquel renvoie " ce germe précieux ", est mort d'une épidémie de rougeole en I712. L'Histoire s'écrit ici sur le mode non plus du mythe, mais de l'occasion manquée et de l'accidentel ${ }^{\mathrm{II}}$ : l'éternuement du héros suffit à "porter le trouble dans le mouvement des tourbillons » royaux (p. IO2)!

Amilec le rassure : ce n'est qu'un germe de Flatteur qui l'aura irrité ; car des graines d'hommes et surtout leurs " germes " " errent partout autour [des hommes] " et leur provoquent toutes sortes de réactions, "suivant le naturel des personnes dont ils sont échappés, et la disposition de celles sur lesquelles ils se trouvent à portée d'agir " (p. I03). Préfiguration des microbes que Tiphaigne a pu tirer de la doctrine alchimique de l'émanation - selon laquelle les " âmes " les plus pures, issues de "l'univers de l'émanation ", suivent un " flux divin vivifiant " jusque dans les corps des humains ${ }^{12}-$, et qu'il fait servir à une " Panacée " modernisée :

Io. Bernardin de Saint-Pierre, Études de la nature, Bâle, I797, t. 5, p. 368.

II. Les textes « utopiques » de Tiphaigne poussent tous les systèmes à l'absurde : voir Giphantie (1760), L'Empire des Zaziris (176I) et L'Histoire naturelle, civile et politique des Galligènes (1765). Le premier est accessible dans Voyages au pays de nulle part, éd. F. Lacassin, Robert Laffont, "Bouquins ", Paris, I990.

I2. Maurice-Ruben Hayoun, art. " émanation [judaïsme] » du Dictionnaire critique de l'ésotérisme, dir. Jean Servier, Paris, PUF, I998, p. 466. 
D’où vient la plupart de ces guérisons inattendues dont le Médecin a soin ${ }^{13}$ de se faire honneur, sans y avoir en rien contribué ? De graines d'hommes. Que ne s'appliquet-on à faire des microscopes assez bons pour les apercevoir à la surface des corps, et des outils assez déliés pour les y recueillir. On y trouverait des ressources sûres contre les maladies les plus opiniâtres. (p. IO3-IO4)

L'alchimie a besoin de microscopes, et c'est peut-être dans cette articulation des savoirs qu'innove le plus Tiphaigne : le progrès des sciences serait à ce prix.

\section{Un cabinet de curiosités}

Car en attendant, comme le fait dire Tiphaigne à Zamar, lieutenant lunaire d'Amilec :

Les faits se multiplient, les expériences s'accumulent, l'esprit s'en étonne et s'y perd, tout est désespéré, à moins qu'il ne survienne quelqu'Amphion qui au son de sa lyre anime ces assemblages informes de matériaux, et construise par enchantement cet édifice tant attendu. (p. 57)

La réussite de l'Encyclopédie ne semble pas ici bien assurée, mais l'éloge de Diderot, dont Tiphaigne partage les vues vitalistes, est évident. Diderot a peut-être lu Tiphaigne, tous deux ont lu Maupertuis, et on peut reconnaître, de l'un à l'autre, les mêmes images - l'essaim d'abeilles, les cornets de papier ${ }^{14}$, l'arbre et les branches, etc.

Le Rêve de d'Alembert note en 1769 : "Tout animal est plus ou moins homme ; tout minéral est plus ou moins plante ; toute plante est plus ou moins animal. " Or, cette nature une et entièrement vivante ne peut qu'évoquer la vision renaissante et alchimique d'un monde en perpétuel mouvement et tissé de correspondances; de fait, nombreux sont les points de passage entre l'alchimie, et en général la science de la Renaissance, et les sciences "modernes ", que ce soit la chimie, la physique ou la biologie. Et ce sont ces complexes rapports, où l'art et la magie se mêlent aux sciences de la matière et du vivant ${ }^{15}$, que Tiphaigne représente sur

13. L'édition Grèges note ici "foin », qui est une coquille.

I4. Un génie apporte à Amilec des graines d'amants dans un "très petit cornet de papier ", et d'Alembert dans son rêve voit une " chambre tapissée de petits cornets, et sur chacun de ces cornets une étiquette : guerriers, magistrats, philosophes, poètes, cornet de courtisans, cornet de catins, cornet de rois" (p. 893).

I5. Voir à ce sujet H. Bredekamp, La Nostalgie de l'antique : statues, machines et cabinets de curiosités, Diderot éditeur, arts et sciences, Paris, New York, Amsterdam, I996, par ex. les p. I2I-I22 sur Bufffon et Linné. 
un mode fictionnel. L'attraction est dans ce domaine un exemple frappant : Tiphaigne emploie les concepts alchimiques de "sympathie » et d' " antipathie ", repensés chez Newton en "attraction " et " répulsion ", pour illustrer la sélection des graines d'auteurs sensés d'une part (p. 35), et la migration des graines "d'Étourdi " sur la Lune d'autre part (p. 45). Autrement dit, pour rendre compte du difficile partage entre la raison et la folie : la fiction représente, autant qu'elle s'en nourrit, l'évolution et la transformation des savoirs. De là sa prédilection pour les fréquents et étonnants points de rencontre entre l'alchimie et la science moderne, telle la description du système solaire ; aussi le voyage d'Amilec suit-il l'ordre alchimique des planètes et celui des métaux correspondants, du Soleil (l'or) jusqu'à Saturne (le plomb) en passant par la Terre, mais est " justifié " par des considérations sur l'âge des planètes qui semblent sorties de la cosmogonie de Buffon : "Mercure étant le plus proche du Soleil, a mûri le premier»(p. 27). Réciproquement pour ainsi dire, c'est Buffon qui semble reprendre des images ésotériques lorsque pour expliquer l'origine des planètes il invoque, comme le cite Tiphaigne, une « éclaboussure du Soleil, qui en saillit ${ }^{16}$ dans le temps qu'une Comète vint maladroitement fondre sur cet Astre " (p. 59) ${ }^{17}$.

Cette paradoxale proximité des théories et représentations est figurée dans la fiction par le contenu hétéroclite du " cabinet de curiosités naturelles " d'Ataman, " marchand de physique en gros et en détail " de la Lune, où on trouve des "fragments de matières ", un " corps d'homme [...] pétrifié ", l'inévitable "Pierre philosophale », des " gas » et autres objets magiques. Le cabinet de curiosités constitue ici un modèle à plusieurs entrées et plusieurs dimensions : au cabinet anthologique d'Ataman répond le très actif laboratoire d'Amilec, dont les méthodes et principes mêlent chimie, physique, botanique, morale et politique. Enfin, c'est tout le texte qui apparaît comme un "cabinet de curiosités ${ }^{18}$ ", en particulier dans sa façon d'articuler une vision « rationnelle » du vivant (autrefois mécaniste, ici plutôt vitaliste) et des procédés et ingrédients magiques. Aussi les boîtes et les tamis avec lesquels Amilec manipule les " graines d'hommes » n’ont-elles rien à envier par exemple aux « boîtes trembleuses » du château

I6. Autre coquille dans Grèges, qui note " faillit ».

17. La cosmogonie de Buffon est ébauchée en 1749 dans son Histoire naturelle et définitivement formulée en 1780 dans les Époques de la nature.

I8. Dont le modèle est celui de Bacon, à la fin du Xvi ${ }^{\mathrm{e}}$ siècle - une bibliothèque, un jardin, un zoo et bien sûr un " laboratoire chimique ". 
$\mathrm{d}^{\prime} A \mathrm{mbras}^{19}$, et le narrateur note à plusieurs reprises son admiration devant l'enchantement qui lui est offert.

La référence est mâtinée d'ironie : chez Ataman, la boîte renfermant la fameuse pierre «n'est visible que de loin : plus on s'en approche, plus elle devient diaphane, et enfin elle disparaît entièrement, dans l'instant même qu'on se croit à portée de la saisir ${ }^{20}$ ". Il peut sembler facile de renoncer à ce qui n'existe pas. Mais que faire des êtres bizarres que fournit l'expérience? "Je n'en finirais pas [...] » dit Zamar, dans une mise en abyme du texte et de toutes ses "suites " possibles ${ }^{21}$. Le voyage dans la Lune est en effet susceptible d'infinies variations, thématiques et littéraires - de Lucien à Kepler ou Cyrano. Le Songe ou astronomie lunaire ${ }^{22}$ de Kepler présente certes des êtres lunaires bien différents de ceux de Tiphaigne - la chaleur régnant sur la Lune oblige tous les êtres à une vie souterraine dont se souviendront Verne et Wells -, mais il imagine aussi des " êtres vivants " sortant de corps calcinés à la tombée de la nuit. Quant à Cyrano, qui a lu le texte de Kepler, il imagine que les êtres nés dans le Soleil, se trouvant trop nombreux, sont venus peupler la Terre et la Lune. La vie extraterrestre a perdu le caractère possiblement hostile qu'elle a chez Kepler et qu'elle retrouvera dans la science-fiction, au profit d'une démultiplication des points de vue intellectuels, scientifiques comme philosophiques, portés sur la vie terrestre - procédé courant au XVIII ${ }^{\mathrm{e}}$ siècle, chez Voltaire comme chez Tiphaigne : Micromégas et son Saturnien, Amilec et son lieutenant Zamar, autant de voix pour parler de l'homme et l'analyser.

L'enchâssement des discours et des savoirs est source de jeu chez Tiphaigne, qui au centre de son livre fait d'Amilec le docile auditeur de Zamar, dont l'explication se conclut par ces mots : "Vous êtes trop bon Physicien, Illustre Amilec, pour ne pas être satisfait de ces raisons » (p. 47). La fiction lunaire inverse les hiérarchies énonciatives et gomme les frontières entre la réalité du héros-narrateur et le merveilleux d'Amilec. Circulation du texte comme des êtres : si Zamar a été envoyé par Amilec, son Courrier reconnaît le narrateur; c'est qu'il a " connu un de ses enfants qui lui ressemble si fort qu'en voyant le père, [il a] cru voir le fils" (p. 62). La mise à distance est aussi rapprochement ; la lune et la terre se confondent, comme peut-être Amilec et l'auteur : une signature orne la troisième

19. Horst Bredekamp, ouvr. cité, p. 62.

20. Amilec, ouvr. cité, p. 60.

2I. De fait, selon la bibliographie utopique d'Hartig et Soboul, Amilec aurait fait l'objet d'une troisième édition, chez Michel Lambert, en 1754, augmentée d'une "Suite de Zamar ".

22. J. Kepler, Le Songe ou astronomie lunaire, [I634], éd. et trad. M. Ducos, P. U. Nancy, 1984. 
édition, "A.D.P ", qu'on traduit généralement en "Ami des Planètes "; or le nom d'Amilec, outre le décryptage alchimique de Fulcanelli, suggère une décomposition en Ami-lec, la syllabe finale jouant le rôle d'indice métatextuel renvoyant au conte oriental.

La fiction lunaire prolonge la satire sociale et généralise la critique de la science contemporaine ; les physiciens sont des "Marchands", soit " en gros " pour les " faiseurs de systèmes ", soit " de détail " pour ceux qui s'occupent « toute leur vie » d'un « rien » - de préférence d'insectes... (p. 53). Leurs ouvrages, intitulés "Recueil de riens », "Grand Dictionnaire Universel " ou "Coup d'œil ", semblent ne se préoccuper que de leur propre forme, et s'ils « font beaucoup de bruit » ce n'est guère à l'honneur de la société qui les $l i t^{23}$ - car la science est à la mode, ce qui serait le sujet implicite d'Amilec autant que sa condition d'existence.

\section{Une galerie plaisante : la science comme merveille}

Sur l'ensemble du texte, Amilec ne se départit pas d'un ton humoristique qui traite avec la même irrévérence les hommes, les "Lunaires " et les " génies ", et quant à l'adresse de Tiphaigne " aux savants ", elle est d'une ironie acide. À l'opposé, Diderot dédie la même année ses Pensées sur l'interprétation de la nature " aux jeunes gens qui se disposent à l'étude de la philosophie naturelle ». Certes, la parole de Tiphaigne n'est pas autorisée, du moins pas autant qu'il le faudrait pour que sa rêverie ait le statut épistémologique de celle de Diderot ; mais surtout Tiphaigne ne se donne pas le même public : en conteur et en littérateur, il commente la société dans laquelle il vit autant que la littérature qu'elle consomme. De ce fait la sexualité, absente de la théorie de la génération présentée par Amilec, sert au fil du texte des allusions plaisantes qui articulent satire sociale et satire du savoir : on plaisante volontiers le savant sur ses expériences. C'est la première scène du songe : Amilec montre à son protégé " cinq ou six génies occupés à recueillir la graine d'hommes »; les trois premiers respectivement travaillent auprès d'un Officier, d'un honnête homme et d'un Gouverneur, et les trois autres "s'occupent " " autour " d'une parmi les "plus belles femmes que l'on puisse voir» (p. 2I) ; comme le souligne le héros : " ce serait dommage d'en perdre un seul germe ». C'est qu'elle

23. Cette critique est explicitée par le prologue des «Papillons » ajouté à la seconde édition, dans lequel le texte est présenté comme le récit d'un songe occasionné par la lecture d'une brochure extravagante (que le narrateur n'aurait pas lue sans le conseil exprès de sa dame). 
est vertueuse, explique Amilec, lequel livre ensuite sa recette pour sélectionner de telles graines :

Je fais jeter dans une boîte pleine de graines féminines huit ou dix germes de PetitMaître : il s'excite aussitôt un mouvement intestin des plus violents. Quand il s'est apaisé, et que tout est en repos, on trouve dans la boîte, autant de gros pelotons, qu'on y avait jeté de germes de Petit-Maître. (p. 22)

Il suffit alors d'ôter ces pelotons et de récolter les graines restantes. On retrouve ici une série de termes à double entente bien faits pour amuser le lecteur. Mais Tiphaigne n'appuie pas ce trait facile, simple indice de la portée ludique du texte. L'intéresse davantage l'inépuisable imagerie des savoirs, dont ces germes partout répandus constituent à la fois un exemple et un modèle. Échappés des pores des hommes ou contenus dans la liqueur séminale, ils évoquent aussi bien le "sperme élémentaire " imaginé par l'alchimiste Sendivogius ${ }^{24}$, vivifié par les rayons célestes, digéré au sein de la terre puis resurgissant à sa surface, que les germes préformés disséminés dans l'air de Hartsoecker ou les molécules organiques de Buffon ${ }^{25}$.

Les références alchimiques étaient bien présentes au XVIII ${ }^{\mathrm{e}}$ siècle, et pas toujours considérées de façon critique : le Dictionnaire de Trévoux comme l'Encyclopédie regorgent d'emprunts et de passages recopiés des différents ouvrages de vulgarisation, de critique et d'histoire alchimiques. Ainsi Jaucourt définit-il la semence comme un "embryon de la graine ", contenant un "baume » capable de retenir « cet esprit subtil, pur et volatil, qui est la plus parfaite production de la plante, et que les Alchymistes appelent esprit recteur, habitant du soufre Archée, serviteur de la nature ". Chez Tiphaigne, toutes ces conceptions deviennent des départs de fiction, comme l'illustre cette sorte de bal occasionné par le jeu d'une basse de viole qui met en scène la vie, la mobilité et la sensibilité des graines d'hommes. Le passage démarque sans doute celui du Voyage dans la lune où Cyrano présente les deux « idiomes » des Lunaires, la musique instrumentale pour les Grands, les «trémoussements ${ }^{26}$ " pour le peuple. L'emprunt à Cyrano

\footnotetext{
24. Voir D. Kahn, article cité.

25. Qui inspirent à Diderot cette jolie déclaration à Sophie Volland : «O ma Sophie [...] si je devais dans la suite des siècles refaire un tout avec vous, si les molécules de votre amant dissous venaient à s'agiter, à se mouvoir et à rechercher les vôtres dans la nature! Laissez-moi cette chimère [...]. » (Lettre de Diderot à Sophie Volland du I5 octobre 1759, dans Correspondance, éd. G. Roth, Paris, Minuit, I956, t. 2, p. 284). Voir J.-L. Fischer, "Buffon et les théories de la génération au $\mathrm{XVIII}^{\mathrm{e}}$ siècle ", La Pluridisciplinarité dans les enseignements scientifiques, t. I : histoire des sciences, Actes de l'université d'été, I6-20 juillet 200I, Poitiers, disponible sur <www.eduscol.com>.

26. Cyrano de Bergerac, L'Autre Monde ou Les États et Empires de la Lune, éd. M. Laugaa, GF Flammarion, Paris, 1970, p. 60.
} 
est signalé par le vocabulaire (les graines "se trémoussent ») et par le trait social, systématisé chez Tiphaigne - le « spectacle » offert aux yeux et aux oreilles délivre " une image de la société humaine " avec ses "différentes passions " (p. 7I). Mais Tiphaigne prête à l'épisode une dimension alchimique, du fait, à la fois, de la réflexion sur l'harmonie du monde ${ }^{27}$, prise ici à contresens, et de la posture des personnages, créant et défaisant à loisir cette harmonie.

Le héros semble avoir atteint dans cet épisode le statut d'adepte : prenant l'instrument et "jou[ant] une contredanse ", le héros "d'un coup d'archet ${ }^{28}$ " peut " mettre en branle des Nations entières ". L'adepte, tel le mage, tel le savant aussi, peut corriger et diriger les hommes - l'" archet " renvoyant peut-être à l'Archée alchimique, origine de toute chose, resté d'ailleurs dans le vocabulaire de la biologie moderne, pour désigner les formes de vie les plus primitives. Mais ici, il s'agit plutôt de mettre le désordre partout, et on a vu qu'un éternuement peut produire le même effet. Les efforts des meilleurs savants ne semblent devoir susciter que le rire ; et c'est sur cette vision plaisante, qui évoque Micromégas, que Tiphaigne ouvre son ouvrage :

Imaginez-vous [le narrateur rapportant son rêve s'adresse au lecteur] un Physicien, qui avec toute l'attention et la sagacité possible, s'occupe à sonder le duvet qu'il aperçoit sur l'aile d'une mouche ; telle était à peu près l'attitude de chacun de ces Génies moissonneurs. Il n'y avait pas moyen de s'empêcher de rire ; j'allais éclater $[\ldots]^{29}$. (p. 20)

Si les naturalistes de l'infiniment petit - la référence aux insectes vise sans doute Réaumur - sont ridiculisés, les « faiseurs de systèmes » ne sont pas plus fiables. Et le premier d'entre eux, dans le texte, c'est Amilec.

\section{Le rêve et la combinatoire : la fiction comme paragramme}

Amilec est un génie, un manipulateur de germes, un mage, un initiateur : un être merveilleux qui répond en songe aux questions d'un savant besogneux et peu doué. Pourtant, la révélation fait long feu et le réveil du narrateur est douloureux :

27. Voir, comme illustration de cet emploi de la musique en alchimie, le traité de R. Fludd par exemple, Monochordum mundi symphoniacum, Francfort, I623 (voir illustration dans Alexander Roob, Alchimie et mystique, le cabinet hermétique, Icons, Taschen, 2005, p. 25).

28. Coquille dans Grèges, qui note " archer ».

29. Voir Voltaire, Micromégas [env. 1739], 1752, chap. 5. 
Mais quel chagrin succéda à cette joie, quand je me retrouvai seul dans mon cabinet, au milieu de mes tristes volumes, et privé peut-être pour toujours de la compagnie d'Amilec! Une jeune femme que d'impitoyables Corsaires enlèvent d'entre les bras d'un Époux chéri, n'est pas atteinte d'une plus vive douleur. Amilec, m'écriai-je, savant Génie, généreux Amilec, pourquoi m’abandonnez-vous? Mais je l'appelais en vain $^{30}$; les Génies Moissonneurs, les Génies Éplucheurs, le Grand-Maître Amilec, tout avait disparu, tout était perdu pour moi. (p. Io6, fin du texte).

Cette fin fait se rejoindre tous les fils d'un pastiche composite : l'épluchage renvoie au feuilletage des opérations des alchimistes, imbriquées les unes dans les autres, sans cesse superposant les " règnes " de la matière, mais il renvoie aussi aux fastidieux travaux des savants. Pastiche littéraire aussi : la déception du narrateur évoque immanquablement celle de la narratrice du Sylphe de Crébillon, et avec elle celle de nombre de personnages des contes des années I730-I75O. Ces déceptions seraient, semble nous dire Tiphaigne, de même nature : solitude, manque, inquiétude, incapacité à distinguer le vrai du faux. Tiphaigne use de l'antiphrase pour abattre la prétention des savants auxquels il s’est épuisé à vouloir ressembler : "Qu'il est doux, qu'il est beau de rêver, quand nos songes peuvent éclairer l'Univers et immortaliser notre nom !» (p. 9)

Or, la même année qu'Amilec, paraît le premier état de ce qui devient l'année suivante les Pensées sur l'interprétation de la nature ${ }^{31}$; le terme "Pensées » est une correction de I754, de même qu'à l'intérieur du texte, celui de "rêveries" est remplacé par " conjectures": Diderot met alors en avant, pour le public, la rigueur de l'observation et du raisonnement. En revanche, dans le cadre d'un texte d'usage sinon privé du moins restreint, il souligne et exploite la productivité du rêve, de ses ressorts et de sa logique, pour la réflexion scientifique : le Rêve de d'Alembert est écrit, semble-t-il, en 1769 , et circule ensuite sans qu'il y ait d'édition officielle ${ }^{32}$. La portée du texte de Tiphaigne est ambivalente : s'il récuse, dans le paratexte, la capacité du rêve et de la science à dire le vrai, Tiphaigne joue dans la fiction sur les deux tableaux de la satire traditionnelle et du conte contemporain. Au « songe philosophique » attendu et annoncé, il associe le

30. Chez Crébillon : "Je l'ai depuis vainement rappelé. " (Le Sylphe, éd. M. Delon, Sylphes et sylphides, anthologie, Desjonquères, Paris, 1999, p. 75)

31. Denis Diderot, Pensées sur linterprétation de la nature, I754 [I753], éd. Colas Duflo, GF Flammarion, Paris, 2005. Voir son appareil critique concernant les différents états du texte, ainsi que les échos aux textes de Buffon et de Maupertuis cités en annexe.

32. Pour ces éléments bibliographiques, voir la notice sur le site de la Société Diderot <www. swif.uniba.it/lei/filmod/testi/diderot.htm> (consulté le I8 mai 2009); pour le texte, voir Le Rêve de d'Alembert et autres écrits philosophiques, préface, commentaires et notes de Jacques et AnneMarie Chouillet, Paris, coll. " Le Livre de poche ", I984. 
songe merveilleux des contes de son temps : le songe déjà chez Aulnoy et plus encore chez Crébillon est lié aux sens et au désir, ce que Tiphaigne rappelle en plaçant ses personnages, quoique en fort sérieuse discussion, sur un "nuage qui semble former un canapé » (p. 72). Souvenir du Sopha: il n'y a pas meilleur lieu pour causer de la génération! Pas de meilleur lieu non plus, dans une irrévérence subtile, pour assister aux tourbillons royaux...

Le mélange des merveilleux met en pratique le principe d'analogie, comme mode de raisonnement, modèle et procédé d'écriture, modalité aussi de fiction. De sorte que l'analogie n'est plus, comme chez Diderot, vectrice d'unité, mais indice de dysfonctionnement : les germes pullulent, et pourtant leur disparition menace ; Amilec sait isoler les germes merveilleux, mais ces germes sont " exténués " - au point qu’il faille " amalgamer " aux germes de qualité des graines de Financier qui " regorge[nt] de suc " (p. 4I) et qui les dénaturent - et quand ils ne le sont pas, ils se détruisent mutuellement : il faut vite ôter l'" auguste germe " du duc de Bourgogne de la boite avant que tout ne " cède ".

Univers en déréliction donc que celui de Tiphaigne, aussi bien sur un plan astronomique que politique. Quant à l'homme, son être paraît tout aussi fragile : un rien le modifie et rien ne semble le définir; le rêveur de Tiphaigne, désemparé, n'atteint pas la tranquille et heureuse découverte de $\mathrm{M}^{\text {lle }}$ de Lespinasse, découvrant l'unité de sa conscience par-delà les modifications perpétuelles de son être. Pour autant, le désordre des êtres n'est peut-être pas une fatalité : Tiphaigne, par la voix d'Amilec, semble en appeler à une réforme en profondeur, à une "régénération" dont le caractère le plus étonnant, en définitive, serait l'absence de norme de référence; hors mystique, hors idéalisation politique, hors programme social, les efforts d'Amilec frappent par leur caractère désordonné. Les génies eux-mêmes finissent par être comparés à des " abeilles " lorsqu'ils se précipitent «par pelotons " pour assister à « l'Élection des Rois» (p. 83). Et Amilec, pour « tirer la vérité du nuage qui l'enveloppait » (p. 9I), ne trouve rien de mieux que d'inviter son protégé à se caler sur ce nuage-canapé ; l'apothéose finale a lieu elle-même au milieu de cette " espèce d'amphithéâtre " faite des nuages assignés à chacun des génies :

L'aire en était fort unie, fort vaste et de figure circulaire. Tout autour d'elle se terminait par de grosses nues qui formaient comme une chaîne de collines. Sur ces collines on avait distribué par groupes de côté et d'autre une multitude innombrable de Génies de toute espèce. Jamais coup d'œil ne fut plus beau ; je crus voir les Cieux ouverts et tous les Dieux de l'Antiquité rassemblés. (p. 92) 
La merveille, dans le texte de Tiphaigne, ce n'est pas la science ou l'alchimie, ou encore les êtres qu'elles appréhendent ou imaginent, mais leur entrelacement et leur perpétuel engendrement, par greffe, bouture ou " fermentation ». L'inventivité, la puissance de suggestion et la plasticité des savoirs constituent davantage qu'un sujet littéraire : elles modèlent l'écriture de la fiction et de la science. "Il ne faut pas que l'esprit s'arrête avec les yeux " demandait Malebranche dans La Recherche de la Vérité. Tiphaigne, près d'un siècle plus tard, constate et explore, avec un humour traversé de pessimisme, l'étendue des vues ainsi ouvertes à la science. Il enregistre, revendique aussi peut-être, le rôle de l'alchimie dans la constitution de nouveaux savoirs, à un moment où nombre de savants s'évertuent à la rejeter dans le monde des chimères. 